\title{
Electron Diffraction Investigation of Gaseous
}

\section{Tetrachloroethylene and Tetraiodoethylene}

\author{
TOR G. ST RA N D \\ Chemistry Department, University of Oslo, Oslo 3, Norway
}

\begin{abstract}
The molecular structures of tetrachloro- and tetraiodo-ethylene have been studied by the gas electron diffraction method. The molecules possess $D_{2 h}$ symmetry, and the structure parameters with standard deviations are for tetrachloroethylene $r_{\mathrm{C}=\mathrm{c}}=1.354(0.003)$, $r_{\mathrm{C}}-\mathrm{Cl}=1.718(0.003) \AA, \angle \mathrm{ClCCl}=115.7(0.3)^{\circ}$, and for tetraiodoethylene $r_{\mathrm{C}=\mathrm{C}}=1.363(0.015), r_{\mathrm{C}-\mathrm{I}}=2.106(0.005) \quad \AA, \angle$ ICI $=$ $114.2(0.5)^{\circ}$.
\end{abstract}

Tn a previous paper, ${ }^{1}$ the molecular structure of tetrabromoethylene as determined by the gas electron diffraction method was reported. In the present paper the results of electron diffraction investigations of tetrachloroethylene and tetraiodoethylene are given. The same theory, method of analysis, and nomenclature as in the earlier paper are applied throughout this work.

\section{STRUCTURE INVESTIGATIONS}

The experimental data for tetrachloroethylene and tetraiodoethylene were collected on the Oslo electron diffraction unit for an accelerating voltage of about $35 \mathrm{keV}$. For tetrachloroethylene the nozzle temperature was $50^{\circ} \mathrm{C}$, and four plates from each of the two camera distances of about $48 \mathrm{~cm}$ and $19 \mathrm{~cm}$ were applied for the structure determination. For tetraiodoethylene, one of the four best plates from the $22 \mathrm{~cm}$ camera distance did not agree very well with the other three, and three plates for each of the three camera distances of about 130, 48, and $22 \mathrm{~cm}$ were selected for the structure analysis. For this molecule the nozzle temperature was $170^{\circ} \mathrm{C}$.

Treating the data the usual way, ${ }^{2}$ four single intensity curves were obtained for tetrachloroethylene ranging from $s=1.5$ to $s=40$ in intervals of $\Delta s=$ $0.25 \AA^{-1}$. The average intensities from the two camera distances were applied between $s=10.25$ and $s=18 \AA^{-1}$. For tetraiodoethylene the three single intensity curves consisted of intensities from $s=1$ to $s=3.5$ for $\Delta s=0.125$ from the $130 \mathrm{~cm}$ plates, from $s=3.75$ to $s=12.25$ for $\Delta s=0.25$ from the

Acta Chem. Scand. 21 (1967) No. 8 
$48 \mathrm{~cm}$ plates, and from $s=12.50$ to $s=26$ for $\Delta s=0.25 \AA^{-1}$ from the $22 \mathrm{~cm}$ plates.

Nonrelativistic partial waves atomic scattering factors for $35 \mathrm{keV}$ electrons were computed..$^{3}$ For carbon and chlorine the calculations were based upon analytical expressions for the HF potential of the atoms, ${ }^{4}$ and for iodine upon both a TFD potential ${ }^{5}$ and upon a relativistic HFS potential. ${ }^{6}$

The tetrachloroethylene data were modified by the function

$$
\varphi(s)=\frac{s}{\left|f^{\prime}\right|_{\mathrm{c}}\left|f^{\prime}\right|_{\mathrm{Cl}}}
$$

and the data for tetraiodoethylene by

$$
\varphi(s)=\frac{s}{\left|f^{\prime}\right|_{\mathrm{C}}\left|f^{\prime}\right|_{\mathbf{I}}^{\mathbf{R}}}
$$

and the corresponding $g_{m n}$ functions $^{1}$ were calculated. In Fig. 1 are shown the two different sets of $g_{m n}$ functions for tetraiodoethylene. $f^{\prime}{ }_{\mathrm{I}}^{\mathrm{R}}$ and $f^{\prime}{ }_{\mathrm{I}}^{\mathrm{TFD}}$ denote the scattering factor for iodine from the relativistic HFS potential and from the TFD potential, respectively.

The numbering of the atoms in the molecules is shown in Fig. 2. Final results of different least squares refinements are given for tetrachloroethylene in Tables 1 and 2, and for tetraiodoethylene in Table 3 . The results in the tables represent stable least squares minima where further iterations do not change the parameters and the standard deviations given in these tables do

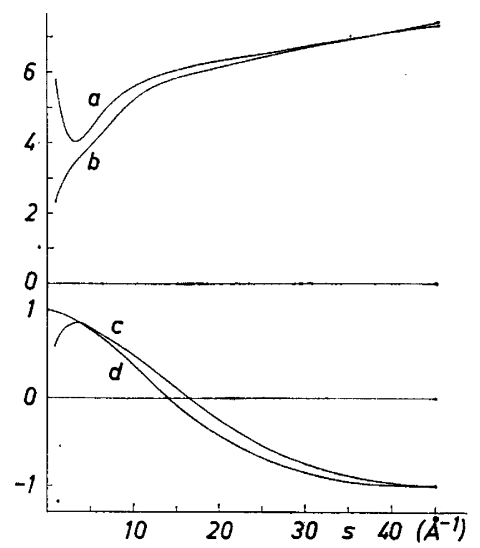

Fig. 1. $g_{m n}$ functions for tetraiodoethylene for a modification function according to eqn. 2. a: $g_{\mathrm{I}-\mathrm{I}}=\left|f_{\mathrm{I}}\right|^{\mathrm{R}} /\left|f_{\mathrm{C}}{ }^{\prime}\right|$, b: $g_{\mathrm{I}-\mathrm{I}}=$ $\left(\left|f_{\mathrm{I}}{ }^{\prime}\right|^{\mathrm{TFD}}\right)^{2} /\left|f_{\mathrm{I}}\right|^{\mathbf{R}}\left|f_{\mathrm{C}^{\prime}}\right|, \mathrm{c:} g_{\mathrm{C}-\mathrm{I}}=\left|f_{\mathrm{I}}\right|^{\mathrm{T}}{ }^{\mathrm{TFD}}$ $\left.\cos \left(\eta_{\mathrm{I}}^{\mathrm{TFD}}-\eta_{\mathrm{C}}\right)|| f_{\mathrm{I}}{ }^{\mathrm{T}}\right|^{\mathrm{R}}$, and $\mathrm{d}: g_{\mathrm{C}-\mathrm{I}}=$ $\cos \left(\eta_{\mathrm{I}}^{\mathrm{R}}-\eta_{\mathrm{C}}\right)$. R and TFD denote scat. tering factors obtained from a relativistic HFS potential and a TFD potential respectively.<smiles>[X]C([Y4])=C([Y4])[Y]</smiles>

Fig. 2. Numbering of the atoms in the molecules. 
Table 1. Least squares results for the structure parameters of tetrachloroethylene from four single intensity curves.

\begin{tabular}{|c|c|c|c|c|c|}
\hline$a$ & $b$ & $b$ & $b$ & $b$ & $c$ \\
\hline$r_{\mathrm{C}_{1}}=\mathrm{C}_{2}$ & $1.3503(0.0042)$ & $1.3500(0.0042)$ & $1.3627(0.0031)$ & $1.3525(0.0034)$ & $1.3539(0.0060)$ \\
\hline$r_{\mathrm{C}_{1}-\mathrm{Cl}_{1}}$ & $1.7203(0.0012)$ & $1.7187(0.0012)$ & $1.7156(0.0008)$ & $1.7182(0.0009)$ & $1.7182(0.0016)$ \\
\hline$r_{\mathrm{C}_{1} \cdots \mathrm{Cl}_{2}}$ & 2.6945 & 2.6931 & 2.6975 & 2.6935 & 2.6947 \\
\hline$r_{\mathrm{Cl}_{1}} \cdots \mathrm{Cl}_{4}$ & $2.9103(0.0022)$ & $2.9069(0.0022)$ & $2.9107(0.0016)$ & $2.9093(0.0019)$ & $2.9093(0.0017)$ \\
\hline$r_{\mathrm{Cl}_{1} \cdots \mathrm{Cl}_{2}}$ & 3.1853 & 3.1846 & 3.1797 & 3.1814 & 3.1828 \\
\hline$r_{\mathrm{Cl}_{1}} \cdots \mathrm{Cl}_{3}$ & 4.3147 & 4.3118 & 4.3108 & 4.3111 & 4.3121 \\
\hline$l_{\mathrm{C}_{1}-\mathrm{Cl}_{1}}$ & $0.0489(0.0017)$ & $0.0462(0.0020)$ & $0.0505(0.0012)$ & $0.0463(0.0013)$ & $0.0480(0.0021)$ \\
\hline$l_{\mathrm{C}_{1}} \cdot$ & $0.0582(0.0027)$ & $0.0553(0.0027)$ & $0.0619(0.0019)$ & $0.0575(0.0022)$ & $0.0582(0.0028)$ \\
\hline$l_{\mathrm{Cl}_{1} \cdots \mathrm{Cl}_{4}}$ & $0.0628(0.0019)$ & $0.0619(0.0022)$ & $0.0663(0.0014)$ & $0.0664(0.0017)$ & $0.0644(0.0023)$ \\
\hline$l_{\mathrm{Cl}_{1} \cdots \mathrm{Cl}_{2}}$ & $0.0969(0.0038)$ & $0.0942(0.0038)$ & $0.0976(0.0027)$ & $0.0960(0.0031)$ & $0.0962(0.0015)$ \\
\hline$l_{\mathrm{Cl}_{1} \cdots \mathrm{Cl}_{3}}$ & $0.0627(0.0028)$ & $0.0642(0.0030)$ & $0.0652(0.0020)$ & $0.0635(0.0023)$ & $0.0639(0.0011)$ \\
\hline$\angle \mathrm{ClCCl}$ & 115.5 & 115.5 & 116.0 & 115.7 & $115.7(0.2)$ \\
\hline & $99.5(1.8)$ & $100.0(1.9)$ & $100.6(1.3)$ & $100.4(1.5)$ & \\
\hline$\sum w \Delta^{2} \times 10^{-5}$ & 3.05 & 3.31 & 1.48 & 2.10 & \\
\hline
\end{tabular}

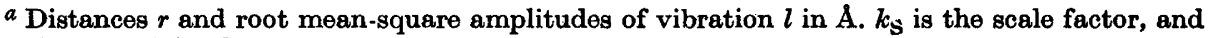
$\sum w \Delta^{2}$ is the weighted square error sum.

${ }^{b}$ Least squares results from four single intensity curves, each from one plate from each of the two camera distances. $r_{\mathrm{C}_{1}}=\mathrm{C}_{2}, r_{\mathrm{C}_{1}-\mathrm{Cl}_{1}}$, and $r_{\mathrm{Cl}_{1}} \cdots \mathrm{Cl}_{4}$ are determined for a planar model of the molecule ( $D_{2 h}$-symmetry) with the other distances as dependent variables. Numbers in paranthesis are uncorrected values for the standard deviations. $w=\exp \left(-0.1(4-s)^{2}\right)$ for $s<4, w=1$ for $4 \leqq 8 \leqq 25$, and $w=\exp \left(-0.006(s-25)^{2}\right)$ for $8>25 \AA^{-1}$.

Average values for the parameters with standard deviations according to eqn. 3 .

not include contribution from systematic errors. Molecular intensity curves are illustrated in Figs. 3 and 4, and radial distribution functions in Figs. 5 and 6 .

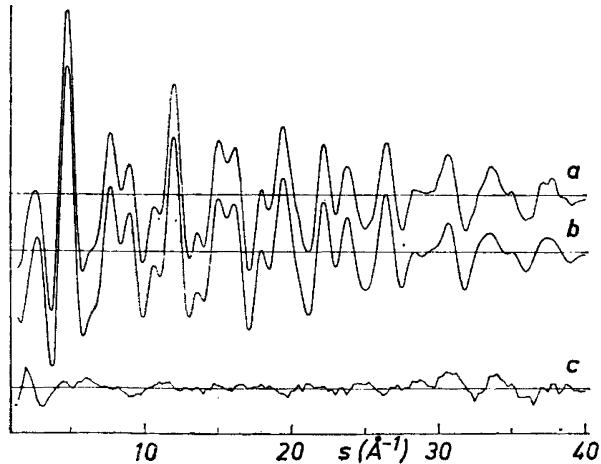

Fig. 3. Molecular intensities for tetrachloroethylene. a: Experimental, b: Calculated from the results of Table 2, b. c: Experimental - calculated.

Acta Chem. Scand. 21 (1967) No. 8

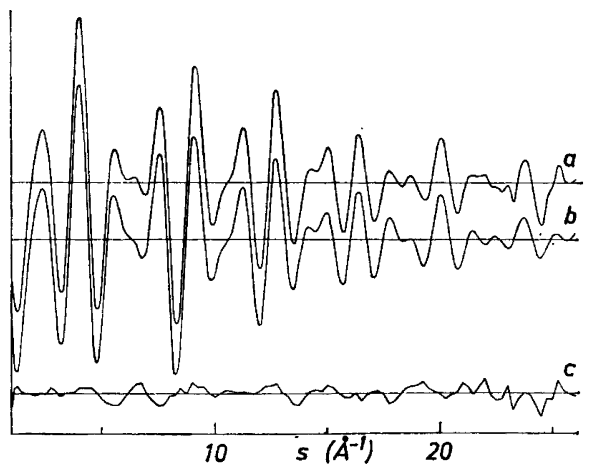

Fig. 4. Molecular intensities for tetraiodoethylene. a: Experimental. b: Calculated from the parameters of Table $3, d$ with scattering factors for a relativistic HFS potential. c: Experimental - calculated. 
Table 2. Least squares results for the structure parameters of tetrachloroethylene from the average of the four single intensity curves.

\begin{tabular}{|c|c|c|c|c|c|c|}
\hline $\boldsymbol{a}$ & $b$ & $c$ & $d / e$ & $f$ & $g$ & $h$ \\
\hline & $1.3536(0.0027)$ & $1.3591(0.0028)$ & & [1.3536] & $1.3546(0.0030)$ & $1.3548(0.0027)$ \\
\hline $\mathrm{Cl}_{1}$ & $1.7182(0.0007)$ & $1.7194(0.0008)$ & & $1.7174(0.0009)$ & $1.7191(0.0007)$ & $1.7168(0.0007)$ \\
\hline${ }_{1} \cdots \mathrm{Cl}_{2}$ & 2.6945 & 2.6959 & 0.004 & $2.6977(0.0020)$ & 2.6961 & 2.6947 \\
\hline $\mathrm{Cl}_{6}$ & $2.9092(0.0014)$ & $2.9089(0.0015)$ & 0.003 & $2.9105(0.0017)$ & $2.9105(0.0015)$ & $2.9056(0.0021)$ \\
\hline $\mathrm{Cl}_{2}$ & 3.1828 & 3.1822 & 0.006 & $3.1824(0.0031)$ & 3.1848 & 3.1842 \\
\hline $\mathrm{Cl}_{2}$ & 4.3120 & 4.3089 & 0.009 & $4.3120(0.0021)$ & 4.3144 & 4.3107 \\
\hline$-\mathrm{Cl}_{1}$ & $0.0480(0.0011)$ & $0.0479(0.0011)$ & 0.0481 & $0.0478(0.0011)$ & $0.0504(0.0017)$ & $0.0415(0.0015)$ \\
\hline$\cdots \mathrm{Cl}_{2}$ & $0.0581(0.0017)$ & $0.0579(0.0018)$ & 0.0578 & $0.0583(0.0017)$ & $0.0693(0.0041)$ & $0.0514(0.0020)$ \\
\hline $\mathrm{Cl}$. & $0.0643(0.0013)$ & $0.0640(0.0013)$ & 0.0672 & $0.0636(0.0013)$ & $0.0726(0.0035)$ & $0.0613(0.0016)$ \\
\hline$l_{\mathrm{Cl}_{1} \cdots \mathrm{Cl}_{2}}$ & $0.0962(0.0024)$ & $0.0963(0.0025)$ & 0.0974 & $0.0956(0.0024)$ & $0.0990(0.0027)$ & $0.0910(0.0035)$ \\
\hline$l_{\mathrm{Cl}_{1} \cdots \mathrm{Cl}_{3}}$ & $0.0639(0.0018)$ & $0.0639(0.0019)$ & 0.0582 & $0.0638(0.0018)$ & $0.0629(0.0021)$ & $0.0599(0.0019)$ \\
\hline$\angle \mathrm{ClCCl}$ & 115.7 & 115.4 & & & 115.7 & 115.6 \\
\hline & $100.1(1.2)$ & $99.9(1.2)$ & & 99. & $101.8(1.0)$ & $85.1(3.2)$ \\
\hline$\sum w \Delta^{2} \times 10^{-5}$ & 1.26 & 1.37 & & 1.23 & 0.54 & 0.76 \\
\hline
\end{tabular}

${ }^{a}$ Distances $r$ and root mean-square amplitudes of vibration $l$ in $\AA . k_{\mathrm{S}}$ is the scale factor, and $\sum w \Delta^{2}$ is the weighted square error sum. Numbers in paranthesis are uncorrected values for the standard deviations, and parameters in square brackets were not varied in the least squares iterations. For all the refinements $l_{\mathrm{C}}=\mathrm{c}$ was fixed on $0.0425 \AA^{?}$.

$b, c, f w=\exp \left(-0.1(4-8)^{2}\right)$ for $s<4, w=1$ for $4 \leqq s \leqq 25$, and $w=\exp \left(-0.006(s-25)^{2}\right)$ for $s>25 \AA^{-1}$.

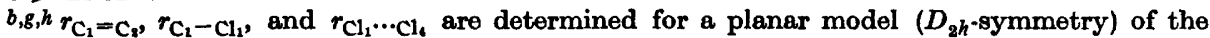
molecule with the other distances as dependent variables.

$c$ Same as for $b$, except that estimated values for the shrinkage effect are included in the model.

$d$ Estimated shrinkage for $c$.

- Root mean-square amplitudes of vibration calculated from spectroscopic data. ${ }^{7}$

$f$ The distances are varied independently with $r_{\mathrm{C}}=\mathrm{c}$ fixed at $1.3536 \AA$.

$g w=\exp \left(-0.1(4-8)^{2}\right)$ for $s<4, w=1$ for $4 \leqq s \leqq 18$, and $w=\exp \left(-0.5(8-18)^{2}\right)$ for $8>18 \AA^{-1}$.

$h^{h} w=\exp \left(-0.5(18-8)^{2}\right)$ for $s<18, w=1$ for $18 \leqq s \leqq 35$, and $w=\exp \left(-0.1(8-35)^{2}\right)$ for $8>35 \AA^{-1}$. $g$ then gives the results for the parameters determined mainly from the intensity data for $4 \leqq s \leqq 18$, and $h$ from the intensity data for $18 \leqq s \leqq 35 \AA^{-1}$.

\section{DISCUSSION}

Tetrachloro- and tetraiodoethylene. The distances in tetrachloroethylene determined for a model with $D_{2 h}$ symmetry are in good agreement with the independently determined distances, and the agreement is not improved when an estimated shrinkage is included in the model (Table 2, $b, c, f$, and $d$ ). The distances obtained only from the data out to $s \simeq 18 \AA^{-1}$ agree very well with the values from the data between $s \simeq 18$ to $s \simeq 35 \AA^{-1}$ (Table $2, g$ and $h$ ). The root mean-square amplitudes from the outer data are smaller than from the inner part, while the values for a more reasonable weighting function compare favourably with the amplitudes calculated from spectroscopic data. ${ }^{7}$

For tetraiodoethylene, the independently determined I...I-distances are in good agreement with the same distances obtained for a model with $D_{2 h}$ symmetry (Table $3, c$ and $d$ ), while the independently determined C-I-distances are somewhat longer than the corresponding distances of the model. 


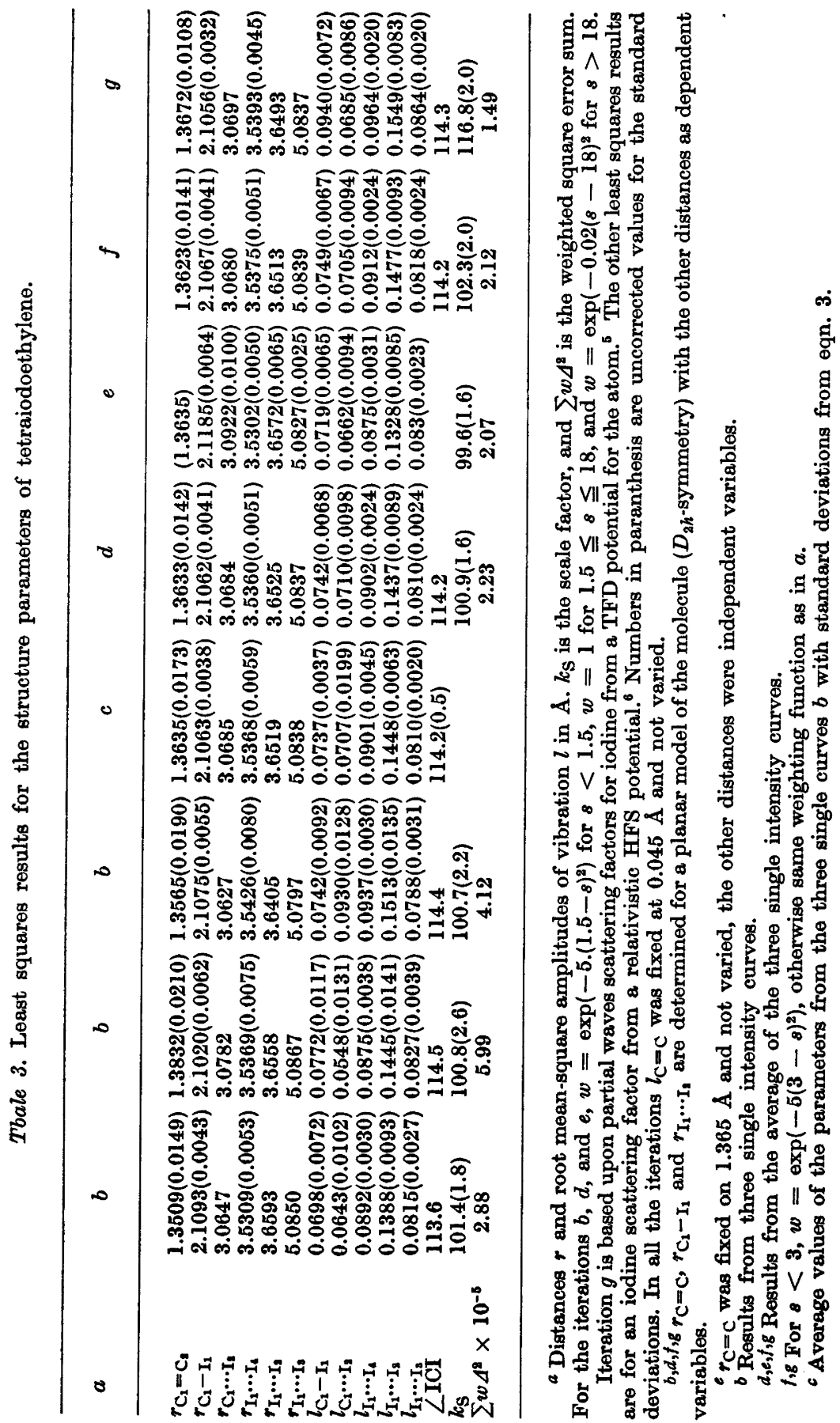

Acta Chem. Scand. 21 (1967) No. 8 


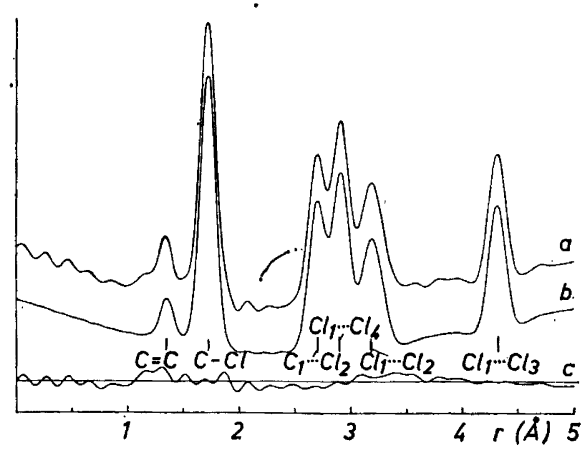

Fig. 5. Radial distribution functions for tetrachloroethylene. A damping function of $\exp \left(-0.0009 s^{2}\right)$ was applied. a: From experimental molecular intensities. b: From calculated molecular intensities. c: Difference.

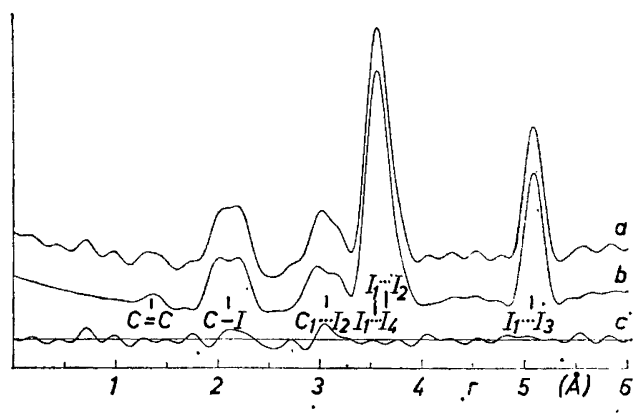

Fig. 6. Radial distribution functions for tetraiodoethylene. A damping function of $\exp \left(-0.0009 s^{2}\right)$ was applied. a: From experimental molecular intensities. b: From calculated molecular intensities. c: Difference.

This would be consistent with a shrinkage effect, but from the function $g_{\mathrm{C}-\mathrm{I}}$ of Fig. 2 it is seen that the $\mathrm{C}-\mathrm{I}$ distances are determined mainly from the intensity data inside $s \simeq 12 \AA^{-1}$, and they would therefore be quite sensible to errors in the data in this range and to errors in the scattering factors.

Table 4. Final results for tetrachloro-, tetrabromo-, and tetraiodoethylene.

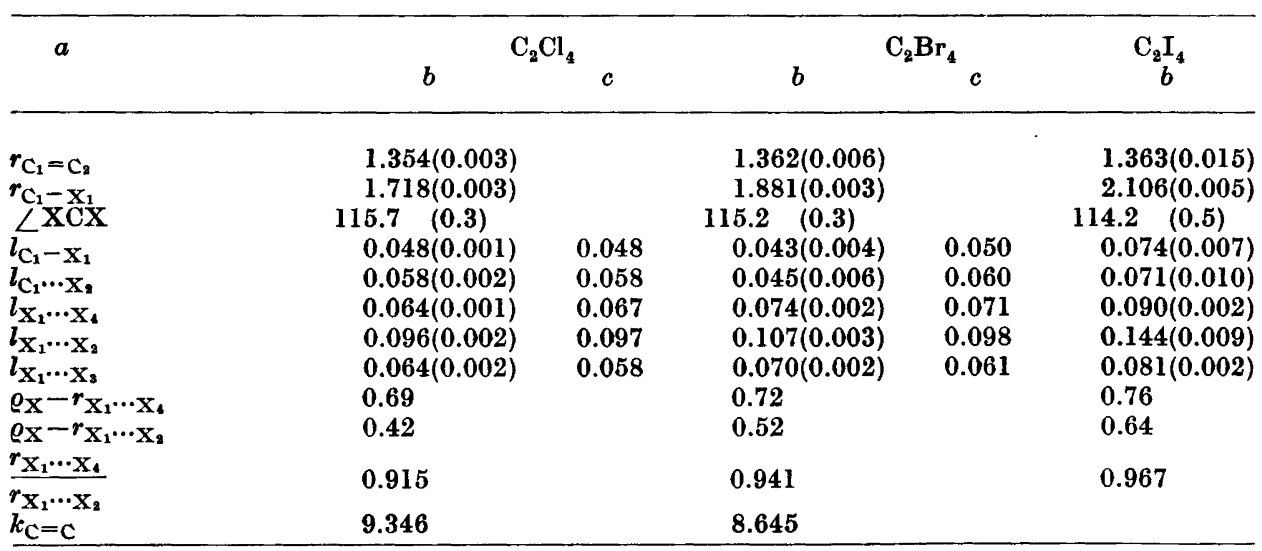

${ }^{a}$ Distances $r$ and root mean-square amplitudes of vibration $l$ in $\AA$. $\varrho_{\mathrm{X}}$ is twice the van der Waals radius for the halogen atom, the applied values are $\varrho_{\mathrm{Cl}}=3.60, \varrho_{\mathrm{Br}}=3.90$, and $\varrho_{\mathrm{I}}=4.30 \AA$. $k_{\mathrm{C}}=\mathrm{C}$ is the $\mathrm{C}=\mathrm{C}$ stretching force constants in mdyne $\AA^{-1}$ from Ref. 7 .

$b$ Results for the structure parameters in the $\mathrm{C}_{2} \mathrm{X}_{4}$ molecules, the results for $\mathrm{C}_{2} \mathrm{Br}_{4}$ are from Ref. 1. Numbers in paranthesis are values for the standard deviations. The standard deviations for the distances include an estimate of contributions from systematic errors, ${ }^{8}$ while the standard deviations for the $l$ 's only include the contribution from random errors.

$c l$-values calculated from spectroscopic data. ${ }^{7}$ 
In Table $3 f$ and $g$ the results for a partial waves scattering factor for iodine from a relativistic HFS potential is compared with the result for a TFD scattering factor, and while the latter scattering factor gives a smaller weighted square error sum, the former one seems to give more reasonable $l$-values, making it difficult to tell from the experimental data which is the better one. In the two refinements, the data inside $s=3 \AA^{-1}$ have been given a very small weight, as the background here is very uncertain, and the present background was drawn to give good agreement when the HFS scattering factor was applied.

In Tables 1,c and 3, , the mean values of the parameters for the two molecules from the results of four and three single intensity curves are given along with standard deviations calculated from the formula

$$
\sigma=\left[\sum_{i=1}^{n}\left(\bar{p}-p_{i}\right)^{2} /(n-1)\right]^{\frac{1}{2}}
$$

where $p_{i}$ is the value obtained in a measurement of a parameter $p$ with a mean value from $n$ measurements of $\bar{p}$. One would not expect to get a very accurate estimate of $\sigma$ from four and three measurements only, but the standard deviations obtained in this way are of the same order of magnitude as the ones obtained from the least squares results for the single intensity curves.

Assuming that the least squares standard deviations for the parameters of the average intensities, $\sigma_{\chi}$, are correct, the standard deviations for the parameters from a single curve should be $\sqrt{n} \sigma_{\chi}$, and if a parameter from the average and single intensities are correlated by a factor $+1 / n$, the standard deviation, $\sigma_{\Delta}$, for the difference $\Delta$ between the average and single least squares parameters are given by

$$
\sigma_{\Delta}=(1+n-2 / \sqrt{n})^{\frac{1}{2}} \sigma_{\chi}
$$

For the $(4+3) \times 8=56$ parameter values from the single curves, $\Delta<\sigma_{\Delta}$ in 46 cases and $\sigma_{\Delta} \leqq \Delta<2 \sigma_{\Delta}$ in 10 cases, indicating that the parameters from the single intensity curves are consistent with the parameters and standard deviations from the average intensities.

Tetra-substituted ethylenes. Final results for the structure parameters of $\mathrm{C}_{2} \mathrm{Cl}_{4}, \mathrm{C}_{2} \mathrm{Br}_{4},{ }^{1}$ and $\mathrm{C}_{2} \mathrm{I}_{4}$ are given in Table 4. The standard deviations for the distances in this table include an estimated contribution from systematic errors in the camera distance and the wavelength of $0.0014 r, 8$ while the

Fig. 7. Badger's rule applied for some $\mathrm{C}_{2} \mathrm{X}_{4}$ molecules. The straight line in the figure has the equation $k^{-1 / 3}=1.26$ $(r-0.98)$ with $k$, the $\mathrm{C}=\mathrm{C}$ stretching force constant, in mdyne $\AA^{-1}$, and the $\mathrm{C}=\mathrm{C}$ bondlength $r$ in $\AA$. The standard deviations for the bondlengths are given.

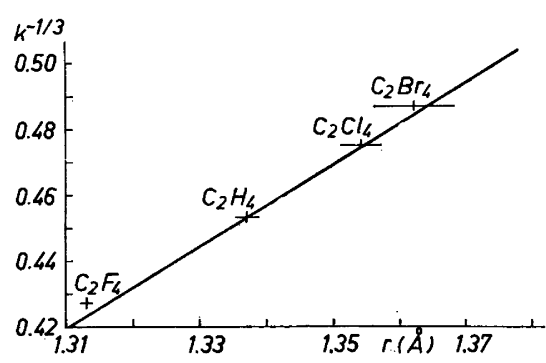

Acta Chem. Scand. 21 (1967) No. 8 
standard deviations for the root mean-square amplitudes are uncorrected values from the least squares refinements. The $\mathrm{C}=\mathrm{C}$ bonds in tetrachloroand tetrabromoethylene seem to be significantly longer than in ethylene (1.337 (0.002) $\AA),{ }^{9}$ and the value for tetrachloroethylene is in good agreement with the electron diffraction value of $1.354(0.005) \AA$, previously determined for cis-dichloroethylene. ${ }^{10}$ The difference between the $\mathrm{C}=\mathrm{C}$ bonds in tetrachloro- and tetrabromoethylene is not significant, however, the variation is in agreement with the variation in the $\mathrm{C}=\mathrm{C}$ stretching force constants for the two molecules. In Fig. 7, Badger's rule ${ }^{11}$ has been applied to correlate the $\mathrm{C}=\mathrm{C}$ stretching force constants ${ }^{7,12}$ with the observed bond lengths for $\mathrm{C}_{2} \mathrm{~F}_{4},{ }^{13} \mathrm{C}_{2} \mathrm{H}_{4},{ }^{9} \mathrm{C}_{2} \mathrm{Cl}_{4}$, and $\mathrm{C}_{2} \mathrm{Br}_{4} \cdot{ }^{1}$

The difference $\varrho_{\mathrm{X}}-r_{\mathrm{X}_{1} \ldots \mathrm{x}_{\mathrm{t}}}$ in Table 4 , where $\varrho_{\mathrm{x}}$ is twice the van der Waals radius for the halogen atom, is for tetrachloroethylene $0.69 \AA$, and this difference increases in steps of about $0.03 \AA$ in going from tetrachloro- to tetraiodoethylene. The difference $\varrho_{\mathrm{X}}-r_{\mathrm{X}_{1} \ldots \mathrm{x}}$ is increasing in steps of about $0.1 \AA$, and as the molecules get more overcrowded, $r_{\mathrm{X}_{1} \ldots \mathrm{X}_{2}}$ gets closer to $r_{\mathrm{X}_{1} \ldots \mathrm{X}_{4}}$. This is demonstrated by the increasing ratio $r_{\mathrm{X}_{1} \ldots \mathrm{X}_{1}} / r_{\mathrm{X}_{1} \ldots \mathrm{X}_{1}}$, and the observed values of the XCX-angles in these molecules must partly be determined by repulsion between the nonbonded halogen atoms.

Acknowledgements. The author wishes to thank The Norwegian Research Council for Science and Humanities for financial support of this work. Thanks are also due to cand.real. A. Almenningen for taking the electron diffraction pictures, to Dr. Jerry A. Peacher for use of the scattering factor computer program, to cand.real. Tor Dahl for the sample of tetraiodoethylene, and to the members of the Oslo electron diffraction group for helpful discussions.

\section{REFERENCES}

1. Strand, T. G. Acta Chem. Scand. 21 (1967) 1033.

2. Bastiansen, O. and Skancke, P. N. Advan. Chem. Phys. 3 (1961) 323.

3. Peacher, J. L. and Wills, J. G. J. Chem. Phys. 46 (1967) 4809.

4. Strand, T. G. and Bonham, R. A. J. Chem. Phys. 40 (1964) 1686.

5. Bonham, R. A. and Strand, T. G. J. Chem. Phys. 39 (1963) 2200.

6. Cox, H. L. Thesis, Indiana University, Bloomington, Indiana 1966.

7. de Alti, G., Galasso, V. and Costa, G. Spectrochim. Acta 21 (1965) 649.

8. Almenningen, A., Bastiansen, O., Haaland, A. and Seip, H. M. Angew. Chem. 77 (1965) 877.

9. Bartell, L. S., Roth, E. A., Hollowell, C. D., Kuchitsu, K. and Young, J. E. J. Chem. Phys. 42 (1965) 2683.

10. Davis, M. I. and Hanson, H. P. J. Phys. Chem. 69 (1965) 4091.

11. Badger, R. M. J. Chem. Phys. 3 (1935) 710.

12. Cyvin, B. N. and Cyvin, S. J. Acta Chem. Scand. 17 (1963) 1831.

13. Karle, I. L. and Karle, J. J. Chem. Phys. 18 (1950) 963.

Received May 3, 1967. 